\title{
Editorial
}

\section{Foodomics: The necessary route to boost quality, safety and bioactivity of foods}

Alejandro Cifuentes

We all know food is essential for our survival. Besides, we all enjoy a tasteful dish and a good beverage. Now imagine we enjoy a safer, higher quality, tastier meal and simultaneously we improve with that meal our health, we enhance our body defenses and fortify our homeostasis. This is the main goal of Foodomics, i.e., to apply $21 \mathrm{st}$ century omics tools and bioinformatics to boost food science investigations, including those related to speed up the resolution of food safety issues, to improve the quality of food and, to understand at a molecular level every claim regarding food and food ingredients bioactivity.

The complexity of the challenge addressed by Foodomics is easy to understand when we consider that, unlike pharmaceuticals, the simultaneous presence of a variety of compounds in a single meal, with diverse chemical structures and concentrations, and with numerous targets with different affinities and specificities increases enormously the difficulty of studying food safety and bioactivity (positive or negative). An additional proof of the complexity of studying food activity on health is to realize that, so far, there are only two works published (one of them from our group) in which the variations at the three levels of expression (transcriptome, proteome and metabolome) were simultaneously interrogated trying to understand the effect

of a given food ingredient. The limitations generated here by the current bioinformatic tools, the lack of information in the databases (e.g. on the identity of many metabolites), our poor knowledge on many molecular processes taking place in cells, or the difficulty to combine the huge data generated by transcriptomics, proteomics and metabolomics approaches (e.g., via systems biology) are still critical here. We need to keep working, probably for a long number of years, before getting the necessary perspective (and knowledge) on these complex and fundamental topics. 
In spite of these limitations, the global outlook that Foodomics proposes is a good response to solve many of the complex issues and drawbacks mentioned above. As a proof of its usefulness, since our first definition of Foodomics in 2009 in a SCI journal, the use of omic approaches in food science and nutrition has quantitatively evolved and grown. The Foodomics concept has also become more and more popular, because it is the ideal frame and distinctive stamp of any work in which an omic approach is used to investigate food safety, quality or bioactivity. As an example of this growth, there are already several Foodomics labs around the world (search e.g., for Foodomics research at CSIC or at RIKILT), there are international conference on Foodomics in Italy (www.foodomics.eu), in France (www.cepia.inra.fr/Evenements/journee-Foodomics-Rennes) in India (www.srmuniv.ac.in/node/10112), videos in YouTube (www.youtube.com/watch?v=U8LBtNilGCs), information in Facebook (www.facebook.com/foodomics2014), in Wikipedia (en.wikipedia. org/wiki/Foodomics), etc. Besides, Foodomics is also getting instruments companies, which now use this term in their presentations and stands.

The interest of the scientific community in modern food analysis and Foodomics and the different trends in this hot area of research are well documented by the present special issue on "Foodomics and Advanced Food Analysis". Namely, this issue presents three review papers dedicated to the structural characterization and bioactivity of oligosaccharides derived from lactose, the study of beneficial plant symbionts for the production of health-promoting phytochemicals and the analysis of biotoxins in food and environment.

A series of original contributions are also presented in this issue, describing the use of several omics and Foodomics strategies to characterize goat milk lactoferrin $\mathrm{N}$-glycans, to obtain the metabolic profile of Vicia faba seeds and blueberries, or to obtain the metabolic profile associated to different rearing conditions in sea bream, to study the bioavailability and bioactivity of antioxidant peptides from soybean conglycinin, to discover human urinary biomarkers of aronia-citrus juice intake, to evaluate protein bio-accessibility in processedmeat upon in vitro digestion, to study the peptide changes in cheese after simulated gastrointestinal digestion or to carry out the targeted metabolomic analysis of resveratrol after acute and chronic intakes of a functional beverage in humans. 
Several original works on the use of CE and LC with different types of detectors (UV, conductivity, laser induced fluorescence, MS) are also presented in this special issue and applied to the analysis of phlorotannins from brown algae Cystoseira abies-marina by comprehensive two-dimensional liquid chromatography, the use of proteins profiles to predict the cultivar of olive leaves and grape cell cultures, the determination of caffeine and taurine in energy drinks, the analysis of steroid hormones in milk, the determination of nucleotides in infant formulas, the classification of Spanish white wines, the analysis and antioxidant potential of phenolic compounds in commercial citrus juices, the phenolic characterization and antimicrobial activity of folk medicinal plant extracts or the effect of rosemary extracts in breast cancer cells. Other original works in this issue demonstrate the possibilities of $2 \mathrm{D}$ microchip-CE to assess the adulteration of dairy products, the use of chip electrophoresis to measure the polyphenols reactivity toward human saliva or the use of microfluidic chips and high-resolution mass spectrometry to investigate the hydrolysis of milk gangliosides by infant-gut associated bifidobacteria.

As editor of this special issue devoted to "Foodomics and Advanced Food Analysis", I would like to thank all the authors for their suitable contributions, all reviewers for the time they devoted to the evaluation of the papers, Prof. Ziad El Rassi for his constant help and support, and to those of Electrophoresis team who contributed with their effort for the preparation of this special issue. 